\title{
Suicide Ideation is Related to Therapeutic Alliance in a Brief Therapy for Attempted Suicide
}

\section{Anja C. Gysin-Maillart PhD, Leila M. Soravia PhD, Armin Gemperli PhD \& Konrad Michel MD}

To cite this article: Anja C. Gysin-Maillart PhD, Leila M. Soravia PhD, Armin Gemperli PhD \& Konrad Michel MD (2016): Suicide Ideation is Related to Therapeutic Alliance in a Brief Therapy for Attempted Suicide, Archives of Suicide Research, DOI: 10.1080/13811118.2016.1162242

To link to this article: $\mathrm{http}: / / d x . d o i . o r g / 10.1080 / 13811118.2016 .1162242$

Accepted author version posted online: 16 Mar 2016.

Submit your article to this journal $\pi$

Џll Article views: 6

View related articles

View Crossmark data ־ 


\title{
Suicide Ideation Is Related to Therapeutic Alliance in a
}

\section{Brief Therapy for Attempted Suicide}

\author{
Anja C. Gysin-Maillart, $\mathrm{PhD}$ \\ University Hospital of Psychiatry, University of Bern, Bern, Switzerland \\ Leila M. Soravia, $\mathrm{PhD}$ \\ University Hospital of Psychiatry, University of Bern, Bern, Switzerland \\ Armin Gemperli, $\mathrm{PhD}$ \\ Department of Health Sciences and Health Policy, University of Luzern, Bern, Switzerland \\ Konrad Michel, MD \\ University Hospital of Psychiatry, University of Bern, Bern, Switzerland \\ Address correspondence to Konrad Michel, MD, University Hospital of Psychiatry, University of \\ Bern, Murtenstrasse 21, 3010 Bern, Switzerland. E-mail: konrad.michel@ spk.unibe.ch
}

\begin{abstract}
Objective: To investigate the role of therapeutic alliance on suicide ideation as outcome measure in a brief therapy for patients who attempted suicide.

Method: Sixty patients received the three-session therapy supplemented by follow-up contact through regular letters. Therapeutic alliance was measured with the Helping Alliance Questionnaire (HAq). Outcome at 6 and 12 months was measured with the Beck Scale for Suicide Ideation (BSS).
\end{abstract}


Results: Therapeutic alliance increased from session one to session three. Higher alliance measures correlated with lower suicidal ideation at 12 months follow-up. A history of previous attempts and depression had a negative affect on therapeutic alliance.

Conclusion: The results suggest that in the treatment of suicidal patients therapeutic alliance may be a moderating factor for reducing suicide ideation.

Keywords: attempted suicide, suicidal ideation, therapeutic alliance, treatment engagement

Individuals who attempt suicide have a high rate of repeated attempts (Owens, Horrocks, $\&$ House, 2002; Sakinofsky, 2000). The repetition rates are highest in the first months after the index attempt (Cedereke \& Ojehagen, 2005; Monti, Cedereke, \& Ojehagen, 2003). In a recent meta-analysis (Carroll, Metcalfe, \& Gunnell, 2014) the estimated 1-year rate of repeated self-harm was $16.3 \%$. Patient-reported rates were higher $(21.9 \%)$ than rates based on hospital records (13.7\%). Suicide attempts by overdosing have a higher rate of reattempts compared to other methods used. In a study from Oslo, nearly $30 \%$ of patients hospitalized for poisoning repeated the poisoning during the first year after the index episode (Heyerdahl et al., 2009). In view of the long-term risk of completed suicide in individuals with a history of attempted suicide (Carroll et al., 2014; Cooper et al., 2005; Jenkins, Hale, Papanastassiou, Crawford, \& Tyrer, 2002) effective interventions for people who have attempted suicide have been given high priority (National Action Alliance: A Prioritized Research Agenda: An Action Plan to Save Lives, 2014). However, up to $50 \%$ of patients do not attend follow-up treatment or drop out of treatment within 1 week (Granboulan, Roudot-Thoraval, Lemerle, \& Alvin, 2001; King, Hovey, \& Brand, 1997; Monti et al., 2003). Self-harm patients who do not receive follow-up treatment have an increased risk of repeated self-harm (Crowder, Van der Putt, Ashby, \& Blewett, 2004). 
So far, only few treatment studies have been able to demonstrate a significant reduction of repeated suicide attempts (Arensman et al., 2001; Brown \& Jager-Hyman, 2014). Exceptions are the studies by Brown et al. (2005), and, more recent, Rudd et al. (2015). However, it appears to be difficult to keep patients in treatment. Brown and colleagues, in up to 24 sessions of cognitive therapy supplemented with various clinical elements introduced case managers to ensure treatment adherence of "no-show" patients and medication compliance. Rudd and co-workers in their cognitive-behavioral therapy of 12 individual sessions offered to active Army personnel, too, used case managers to track participants and to assist with follow-up assessments. Rather surprisingly, some studies found that minimal outreach interventions after attempted suicide, such as contacting patients with regular postcards or standardized letters, are associated with a reduction of suicides (Carter, Clover, Whyte, Dawson, \& D'Este, 2007, 2013; Motto \& Bostrom, 2001), but findings have not been consistent (Beautrais, Gibb, Faulkner, Fergusson, \& Mulder, 2010; Kapur et al., 2013).

A major barrier to the effective treatment of suicide attempters is poor treatment engagement, defined as commitment in the therapeutic process and active participation in a collaborative relationship between therapist and patient (Lizardi \& Stanley, 2010). One possible reason is that suicidal patients often do not feel understood by health professionals, and that the focus of treatment is not relevant for them (Michel, Valach, \& Waeber, 1994; Treolar \& Pinfold, 1993). Clinicians tend to see suicide as a symptom of mental disorder (Jobes, 2000), while for the suicidal individual the central issue is the very personal experience of pain, anguish, hopelessness, and loss of self-esteem (Maltsberger, 2004; Shneidman, 1993). Suicidality is a highly individual phenomenon, with a strong biographical background, and several authors have argued that, in order to increase therapeutic alliance, treatment approaches should be patient-centered and 
collaborative (Lizardi \& Stanley, 2010; Michel, Dey, Stadler, \& Valach, 2004; Michel et al., 2002; Rogers \& Soyka, 2004).

Therapeutic alliance is a consistent predictor of outcome in psychotherapy (Arnow \& Steidtmann, 2014; Horvath, Del Re, Flückiger, \& Symonds, 2011) and is therefore a key element of treatment engagement. It has been characterized as “...the active and purposeful collaboration between patient and therapist" (Gaston, Thompson, Gallagher, Cournoyer, \& Gagnon, 1998). A recent meta-analysis including 190 studies found a significant correlation of $r=.28$ between the alliance and psychotherapy outcome (Horvath et al., 2011). The effect of alliance is increased when the treatment protocol is more structured, and when patients indicate high agreement with the therapist on goals and tasks (Arnow et al., 2014). Horvath, Gaston, and Luborsky (1993) distinguished three universal aspects of therapeutic alliance: (a) The patient's perception that the interventions offered are both relevant and potent; (b) congruence between the patient's and the therapist's expectations of the short- and medium-term goals of therapy; (c) the patient's ability to forge a personal bond with the therapist, and the therapist's ability to appear as a caring, sensitive, and sympathetic helping figure.

We found only one treatment study with suicidal patients investigating the effect of alliance on therapy outcome. Bryan et al. (2012) used the Therapeutic Bond Scale to assess patient-rated evaluation of the treatment relationship, but found no association with the one suicide related item in the Behavior Health Measure-20 as outcome measure. In an earlier study we had reported that in a single assessment interview with patients who had attempted suicide, patient-rated therapeutic alliance measured with the Penn Helping Alliance Questionnaire HAq (Alexander \& Luborsky, 1986) was increased when the interviewer used a narrative interviewing technique (Michel et al., 2004), based on a model of suicidal behavior as a goal-directed action (Michel \& Valach, 1997). 
This approach stands in contrast to the traditional medical model, in which suicidal behavior is usually seen as a symptom of psychiatric disorder. Central to an action theoretical model is the notion that actions are understood as being carried out by agents, that is by persons who are able to monitor their thoughts, emotions, and actions, and who have conscious access to their reasons why they act in such a way. In a typical narrative interview the interviewee is considered the expert of his or her story. The interviewer's primary task is to be an attentive listener and to facilitate the patient's narrative.

We therefore hypothesized (1) that a therapy using a patient-centered, collaborative approach would enhance therapeutic alliance, and (2) that a stronger alliance would be associated with better therapy outcome. Based on a model of suicidal behavior as goal-oriented action we developed ASSIP (Attempted Suicide Short Intervention Program), a highly structured brief therapy consisting of three sessions, followed by 2 years' contact with regular letters (Michel \& Gysin-Maillart, 2015). The findings of the earlier study focusing on therapeutic alliance and narrative interviewing (Michel et al., 2004) prompted us to use a narrative interviewing style, which we complemented with a video playback session (Young \& Valach, 2002). A major focus of ASSIP lies in the development of an early therapeutic alliance, combined with psychoeducation, a cognitive case conceptualization, safety planning, and continued long-term outreach contact.

\section{METHODS}

\section{Procedure and Participants}

The study group represents the treatment arm of a randomized controlled trial on the effectiveness of ASSIP in reducing suicidal behavior. The objective of the present study was to 
investigate if the quality of early therapeutic alliance in ASSIP was related to outcome during a 12-months follow-up period. Suicidal ideation was used as outcome measure.

The study was conducted in a naturalistic setting, with patients being assessed by the duty psychiatrist in the emergency department of the University General Hospital. Psychiatric consultation service was provided by the medical staff of the University Hospital of Psychiatry Berne. The duty psychiatrist informed patients about the study and asked for their written consent to be contacted by an ASSIP therapist. The term "attempted suicide" is used according to Silverman, Berman, Sanddal, O'Carroll, and Joiner (2007, p. 273), i.e., a "self-inflicted, potentially injurious behavior with a nonfatal outcome for which there is evidence (either explicit or implicit) of intent to die". In German speaking countries, similar to North America, the term attempted suicide does not include habitual self-harm, which is characteristic for borderline personality disorders. At the time of contact by the research team the majority of patients had been admitted to the psychiatric hospital or the crisis intervention unit. The responsible clinician was contacted before patients were given an appointment in order to assure the safety of patients for transport to the outpatients department. Patients who had been discharged after medical treatment were contacted directly and offered treatment. Because at the University Hospital of Psychiatry ASSIP was recommended to suicide attempters as the usual procedure, and over $90 \%$ of the patients who fulfilled the inclusion criteria could be allocated to the study. Exclusion criteria for allocation to the study were language problems, current psychotic disorder, and dissocial personality disorders. Clinical diagnoses were made by the duty psychiatrists of the University General Hospital and the University Hospital of Psychiatry, and were based on the ICD-10 Classification of Mental and Behavioral Disorders (WHO, 2014). Written informed consent was obtained from all participants, as well as written agreement for video-recording the narrative interview. Participants completed a 
set of questionnaires after the first therapy session. For the follow-up evaluation participants received the same questionnaires after 6 and 12 months. Study procedures were approved by the local ethics committee in accordance with the principles of the Declaration of Helsinki (Rickham, 1964).

\section{Therapists}

Treatment was provided by four ASSIP-trained therapists. Two of them (KM, psychiatrist; AGM, clinical psychologist and head of special clinic for suicidal patients) were experienced clinicians, while two others (MM and SB, clinical psychologists) with an average of 2 years of clinical practice after graduation were introduced to ASSIP and to the study procedures during the study. Training of the ASSIP protocol including case supervision with the use of the videorecorded narrative interviews was conducted by AGM and KM. The clear structure of the three therapy sessions (see below) provided a strict frame with tasks to be completed in each session, securing adherence to the treatment protocol.

\section{ASSIP Therapy}

ASSIP brief therapy for patients who have attempted suicide is administered in three 6090 min sessions, ideally within 3 weeks. A fourth session can be added if considered necessary. For details see Michel and Gysin-Maillart (2015).

First session: A narrative interview in which the patient is encouraged to tell the story behind the suicide attempt is conducted. The aim of the narrative interview is to reach a shared understanding of the individual mechanism leading to suicidal behavior in a biographical context, and to elicit specific vulnerabilities and trigger events. The interview is video-recorded. 
Second session: Sequences of the video-recorded interview considered to be relevant for further elaboration are selected and viewed on a screen, with patient and therapist sitting next to each other, side by side. The aim of the video-playback is the reactivation of the mental state in the suicidal crisis in a safe environment and the reconstruction of the transition from a mental pain experience to the suicide action. Automatic thoughts, emotions, physiological changes, and contingent behavior are identified. At the end of the session, patients are given a psychoeducative handout, which they are asked to read and comment in writing, and bring back to the next session. After the session the therapist prepares a draft case conceptualization, which includes a formulation of the individual vulnerability in the context of the patient's biography and the specific trigger event(s).

Third session: The patient's feedback to the handout is discussed. The written case conceptualization is revised together with the patient. A list of long-term goals, individual warning signs and safety strategies developed in a collaborative way. Summary and safety strategies are printed and handed to the patient, with copies for the patient's therapist and other relevant mental health professionals. The safety strategies are copy-pasted to a credit-card size "leporello" (a folded leaflet) and handed to the patient with the instruction to keep it the wallet. In addition, patients are given a crisis card with emergency phone numbers.

Continued outreach contact: Patients are sent letters over a time span of 2 years, every 3 months in the first year, and every 6 months in the second year. The letters invite patients to give feedback about how things were going. The content is standardized, with an added remark referring to the personal safety plans developed in the therapy sessions. Letters are personally signed by the ASSIP therapist. If the therapist receives feedback from the patients via e-mail or letters no further re-connection is made until the subsequent letter, unless patients appear to be in a suicidal crisis, 
in which case the therapist contacts them. Any information received from the patient is usually referred to in the subsequent letter.

\section{Measures}

Penn Helping Alliance Questionnaire (HAq). The 11-item self-rating questionnaire HAq (Alexander \& Luborsky, 1986) is used to evaluate the quality of the patient-therapist relationship. The HAq has a good validity for predicting outcome of psychotherapy (Fenton, Cecero, Nich, Frankforter, \& Carroll, 2001). In the present study the German version by Bassler, Potratz, and Krauthauser (1995) is used. These authors found a satisfactory construct validity of the German HAq version in a study involving 239 patients in a 12-week therapy.

The Beck Depression Inventory (BDI) is a 21-question multiple-choice self-report inventory for measuring the severity of depression (Beck \& Steer, 1987). In the present study the German version of the BDI I was used (Hautzinger, Bailer, Worall, \& Keller, 1994).

The Beck Scale for Suicide Ideation (BSS) is a 21-item self-report instrument for measuring the current intensity of the patients' specific attitudes, behaviors, and plans related to suicidal behavior during the past week (Beck \& Steer, 1991). Internal reliability, test-retest stability and validity for the BSS have been established (Brown, 2001; de Beurs, Fokkema, de Groot, de Keijser, \& Kerkhof, 2015). The severity of suicidal ideation is calculated by summing the ratings of the first 19 items. Item 20 (prior suicide attempts) and 21 (severity of the suicide attempt) are not included in the score. The sum scores range from 0 to 38 points. In the present study the German version of the BSS was used (Fidy, 2008).

\section{Statistical Analysis}


Data were analyzed using SPSS version 19.0 statistical software package. Sociodemographic data and outcome measures were examined using $t$-test or chi-square tests. Changes over time in alliance, suicidal ideation and depression, were measured with paired t-tests. In order to elucidate which factors most strongly predict higher suicide ideation over the course of one year, we performed a stepwise multiple linear regression analysis using the HAq, the BDI, and a history of previous suicide attempts (PSA) as independent variables. Where the Mauchly test of sphericity indicated heterogeneity of covariance, we verified repeated-measures results with Greenhouse-Geisser corrections. All tests were two-tailed and a probability of false rejections $<0.05$ was considered statistically significant. All variables were considered normally distributed (Kolmogorov-Smirnov test: $p>0.1$, for all variables).

Missing data: In order to complete the follow-up data hospital records were searched and involved health professionals were contacted personally. The Last Observation Carried Forward (LOCF) method was used to handle missing data. For each individual, missing data were replaced by the last observed value of that variable.

\section{RESULTS}

\section{Participant Characteristics}

Demographic characteristics, psychiatric diagnosis, and history of previous suicide attempts for participants are shown in Table 1. Of the 60 participants, 24 (40\%) were male (mean age 39.42, SD 15.25), and 36 (60\%) female (mean age 34.58, SD 13.42). Thirty-three participants (55\%) were diagnosed with major depression, 8 (13\%) fulfilled the criteria for personality disorder, $17(28 \%)$ for acute stress reaction, and $2(3 \%)$ for substance abuse. Overdosing was the most frequent method used (63.3\%), while lethal methods, such as hanging, shooting, and drowning 
represented $11.7 \%$ of all methods. Twenty-seven (45\%) participants reported previous suicide attempts, 10 of them were multiple repeaters. Three participants (5\%) dropped out within the first 12 months of the follow-up. The reasons were: Psychotic disorder (1), refusal to return the followup questionnaires due to a change of ASSIP therapist (2). The numbers of study participants with complete follow-up data were, at 6 months $n=44(73 \%)$, and at 12 months $n=36(60 \%)$.

\section{Therapeutic Alliance at First and Third Session}

Initial analysis employing paired $t$-test revealed a significant increase in the ratings of the Penn Helping Alliance Questionnaire from the first therapy session (HAq1) to the third session (HAq2) (HAq1: $4.82 \pm 0.6$; HAQ2: $\left.5.19 \pm 0.6, t_{59}=-4.854 ; p=.001\right)$. Bivariate correlations between patient-rated quality of therapeutic alliance and previous suicide attempts revealed a negative association at the first session $(r=-0.34 ; p=.008)$ but not at the third session $(r=-0.13$; $p=.340)$. Thus, a history of previous suicide attempts had a negative effect on the formation of a therapeutic alliance in the first session only. Further analysis revealed a negative association between depression scores and the quality of therapeutic alliance at the first (HAq1: $r=-0.37$; $p=.003)$ and third session (HAq2: $r=-0.27 ; p=.036)$.

\section{Outcome Measure: Suicidal Ideation}

Results of dependent $t$-test revealed a significant decrease in the scores of suicidal ideation from baseline $t 1$ (BSS: $0.39 \pm 0.45)$, to $t 2$ at 6 months (BSS: $0.30 \pm 0.5 ; t_{59}=2.185 ; p=.033$ ), and to $t 3$ at 12 months (BSS: $0.25 \pm 0.44 ; t_{59}=2.674 ; p=.010$ ).

Bivariate correlations (Table 2) between patient-rated quality of therapeutic alliance (HAq1 \& HAq2) and suicidal ideation at 6 (BSSt2) and 12-month (BSSt3) show a significant 
negative association, i.e. higher ratings of therapeutic alliance at the first and third session were associated with lower suicidal ideation at 6 and 12 months. Baseline depressive symptoms (BDI $t$ ) and previous suicide attempts (PSA) correlated with higher suicidal ideation (BSSt3) at one year follow-up.

\section{Predictors for Suicidal Ideation}

Based on the association between therapeutic alliance, depressive symptoms and suicidal ideation, three multiple linear regressions were conducted to analyze the influence of each factor separately: therapeutic alliance, baseline depression, and previous suicide attempts as predictor variables on suicidal ideation after one year as the dependent variable. First session therapeutic alliance (HAq1) accounted for $49.6 \%$ of the variance of suicidality $\left(R^{2}=.496 ; p=.001\right)$, whereas depression $(\mathrm{BDI} t 1)$, accounted for $40 \%\left(R^{2}=.40 ; p=.002\right)$ of the variance. Previous suicide attempts (PSA) explained $55 \%$ of the variance $\left(R^{2}=.555 ; p=.001\right)$.

To overcome the problem of multicollinearity we conducted a linear regression analysis with the correlated baseline measures previous suicide attempt (PSA) and depression at baseline (BDI 1 ) as control variables in step one, and therapeutic alliance in step two (see Table 3). PSA and BDIt 1 explained $30.1 \%$ of the variance. In step two, $8.5 \%$ of variation was added by the variable therapeutic alliance.

\section{DISCUSSION}

The present study investigated the effect of patient-rated therapeutic alliance in the Attempted Suicide Short Intervention Program ASSIP, a specific, highly structured brief therapy for attempted suicide, on the course of suicidal ideation after 6 and 12 months. We had hypothesized that a patient-centered, collaborative therapeutic approach would enhance 
therapeutic alliance, and that patient-rated alliance would correlate with lower suicidal ideation at follow-up.

The main findings in the present study are:

(1) A robust negative correlation between the patient-rated quality of the therapeutic alliance in the first and third treatment session with suicidal ideation in the BSS after 6 and 12 months, i.e. a stronger alliance was associated with lower suicidal ideation after 6 and 12 months. Obviously, therapeutic alliance is determined by what both individuals, therapist and patient, contribute to the therapeutic process. However, it is important to note that the HAq expresses the patients' own evaluation of how much they felt understood and supported by the therapist.

(2) An increase in the scores of therapeutic alliance in the ASSIP group between session one and session three. This is consistent with results from psychotherapy research, which suggest that ratings of alliance in the third therapy session are a good predictor of adherence to treatment and outcome (Saltzman, Luetgert, Roth, Creaser, \& Howard, 1976). We therefore assume that ASSIP therapists were successful in creating a growing working relationship in the course of three sessions.

(3) A negative effect of a history of previous attempts on the quality of the therapeutic alliance in the first session. This effect disappeared in the third therapy session. We therefore assume that an initially skeptical attitude of these participants gave room to a more positive attitude during the following sessions.

(4) A negative effect of depressive symptoms on therapeutic alliance in the first and the third session. This is not surprising, considering that participants with severe depression often 
find it difficult to establish a therapeutic alliance early in treatment. Furthermore, higher baseline depression correlated with more suicidal ideation after 6 and after 12 months.

(5) Regression analysis including therapeutic alliance, previous suicide attempts, and depression revealed that a history of previous attempts had the strongest effect on outcome. This is consistent with findings by Haw, Bergen, Casey, and Hawton (2007). Regression analysis suggests that therapeutic alliance and previous suicide attempts may directly influence outcome, while depression seems to have a negative effect on both.

In summary, ASSIP, although a brief therapy, appears to initiate a therapeutic interaction, increasing therapeutic alliance from session one to three, with depression and previous suicide attempts acting as confounding factors for suicidal ideation as therapy outcome. In our view it is unlikely that the correlation between alliance and outcome is merely the expression of high alliance measures being a marker for low suicidality. Most of our study participants had overdosed. The distribution of methods for attempting suicide is in line with other studies (Horrocks, Price, House, \& Owens, 2003). Overdosing has been associated with higher rates of repeat attempts (Arensman, Corcoran, \& Fitzgerald, 2011), while high lethality methods have a higher risk of subsequent suicide (Bergen, Hawton, Waters, Cooper, \& Kapur, 2010; Horesh, Levi, \& Apter, 2012; Runeson, Tidemalm, Dahlin, Lichtenstein, \& Langstrom, 2010).

We can only speculate on the therapy process factors involved. ASSIP has been devised as a therapy focusing on a patient-centered collaboration. We believe that a strength of the ASSIP protocol are the key elements designed to promote "...the active and purposeful collaboration between patient and therapist" (Gaston et al., 1998). As reported in an earlier study (Michel et al., 2004) a narrative interviewing style was associated with better therapeutic alliance. We believe that the initial narrative interview may set the basis for a collaborative working alliance. In addition, 
ASSIP includes various therapeutic elements (video-playback, psychoeducation, safety planning, personalized regular letters), which are all aimed at strengthening the collaborative therapeutic relationship. The finding that the scores of therapeutic alliance increased from session one (HAq1) to session three (HAq2) is consistent with results from psychotherapy research, which found that ratings of alliance in the third therapy session are a good predictor of adherence to treatment and outcome (Saltzman et al., 1976).

The patient information about the limited therapy goals may have been a further factor enhancing treatment engagement. Patients were informed that they had an increased risk of future suicidal crises and that the therapy would not cure them from suicidality, but that the aim was to reduce the risk of further suicidal behavior. Furthermore, we assume that the regular letters may have acted as reminders of the increased suicide risk, the warning signs, and the safety strategies developed in the therapy sessions. To our knowledge, so far, no published treatment studies have combined psychological interventions with subsequent ongoing contact through letters.

There are a number of limitations inherent in this study. The Penn Helping Alliance Questionnaire was originally developed to assess therapeutic alliance for psychotherapy with more than 25 sessions (Morgan, Luborsky, Crits-Christoph, Curtis, \& Solomon, 1982), and not for brief interventions such as ASSIP. Luborsky et al. (1996) have developed an improved version of the HAq, which, however, focused even more on long-term therapeutic processes. We explained to the participants that, even if they had an ongoing therapy elsewhere, they should only indicate how the felt after the interview with the ASSIP therapist. The increase of alliance after three sessions found in this study is consistent with results from psychotherapy research, supporting the validity of the HAq ratings. 
Because of the clinical nature of the treatment study we felt that the first session should start with the therapists inviting the patients to tell their story. Therefore the questionnaires were completed immediately after the conclusion of the first session. It could be argued that this poses a problem to the baseline data. However, if the baseline was indeed biased by this procedure, it is most likely that the experience of feeling understood by a caring therapist would have a positive effect on the scores of the baseline measures (suicide ideation and levels of depression), and would therefore reduce the treatment effect.

Missing data regarding the BSS were caused by non-response of participants during follow-up and are a frequent problem of longitudinal studies. The Last Observation Carried Forward (LOCF) method allows to examine trends over time, but tends to underestimate the variability of results, in this case the BSS scores at 6 and 12 months. In view of the decrease of the BSS scores during follow-up LOCF would be expected to underestimate the effect of alliance on outcome. We therefore consider LOCF an acceptable method for our study. Missing data did not concern HAq ratings, which were collected immediately after session one and three.

Therapist continuity was a problem due to the maternity leave of the main investigator (AGM): A considerable number of participants were seen by one therapist but received letters including the follow-up questionnaires signed by a different person. This is a practical problem typical for real-world treatment studies, which must be expected to reduce treatment effects.

It may be questioned how much a decrease of suicidal ideation correlates with lower risk of further suicidal behavior. The BSS suicide ideation items are composed of three factors: Desire for Death, Preparation for Suicide and Actual Suicide Desire (Steer, Rissmiller, Ranieri, \& Beck, 1993). A high proportion of people indicating suicide plans (72\%) move from plan to attempt (Kessler, Borges, \& Walters, 1999). Suicide ideation in patients who attempted suicide has been 
associated with persistent suicidal behavior (Pinninti, Steer, Rissmiller, Nelson, \& Beck, 2002).

Furthermore, patients with suicide ideation and patients who attempt suicide do not differ in a number of variables generally known to be risk factors for suicide (Pompili et al., 2014). Therefore, BSS scores can be taken as an indirect proxy for actual suicide risk.

The therapists in our study were not involved in the clinical management of the patients. It is possible that this factor had a positive effect on the development of the therapeutic relationship. It will be interesting to see if our findings can be replicated in other clinical settings. Furthermore, although the ASSIP therapy protocol is highly structured, setting clear tasks for each session, we do not yet know how much specific training is necessary for therapists to apply ASSIP. So far, from our experience of training therapists we have found that both, conducting a narrative interview, and formulating a patient-centered case conceptualization, are elements that particularly need close supervision.

In conclusion, the results suggest that therapeutic alliance may have a moderating effect on outcome in the treatment of suicidal patients. To further clarify the role of the therapeutic relationship (and treatment engagement) future treatment studies should include measures of alliance. Patient-centered models of suicidal behavior and collaborative treatment approaches may be particularly promising. Special attention should be paid to patients with a history of attempted suicide and those with a depressive disorder. These individuals appear to have more difficulties in establishing a therapeutic alliance with the therapist.

\section{Acknowledgements}

We thank Millie Megert, who replaced A.G-M. during her maternity leave, and Salome Bühler, who contributed with an ASSIP case. 


\section{References}

Alexander, L. B., \& Luborsky, L. (1986). The Penn helping alliance scales. In L. S. Greenberg \& W. M. Pinsoff (Eds.), The psychotherapeutic process: A research handbook (pp. 325-366). New York: Guilford Press.

Arensman, E., Corcoran, P., \& Fitzgerald, A. P. (2011). Deliberate self-harm: Extent of the problem and prediction of repetition. In R. O'Connor, S. Platt, \& J. Gordon (Eds.), International handbook of suicide prevention (pp. 122). Chichester: Wiley \& Sons.

Arensman, E., Townsend, E., Hawton, K., Bremner, S., Feldman, E., Goldney, R., ..., \& Traskman-Bendz, L. (2001). Psychosocial and pharmacological treatment of patients following deliberate self-harm: The methodological issues involved in evaluating effectiveness. Suicide and Life-Threatening Behavior, 31(2), 169-180. doi:10.1521/suli.31.2.169.21516

Arnow, B. A., \& Steidtmann, D. (2014). Harnessing the potential of the therapeutic alliance. World Psychiatry, 13(3), 238-240. doi:10.1002/wps.20147

Arnow, B. A., Steidtmann, D., Blasey, C., Manber, R., Constantino, M. J., Klein, D. N.,..., \& Kocsis, J. H. (2014). The relationship between the therapeutic alliance and treatment outcome in two distinct psychotherapies for chronic depression. Journal of Consulting and Clinical Psychology, 81(4), 627-638. doi:10.1037/a0031530

Bassler, M., Potratz, B., \& Krauthauser, H. (1995). Der helping alliance questionnaire (HAq) von luborsky. möglichkeiten zur evaluation des therapeutischen prozesses von stationärer psychotherapie. Psychotherapeut, 40, 23-32.

Beautrais, A. L., Gibb, S. J., Faulkner, A., Fergusson, D. M., \& Mulder, R. T. (2010). Postcard intervention for repeat self-harm: Randomised controlled trial. British Journal of Psychiatry, 197(1), 55-60. doi:10.1192/bjp.bp.109.075754

Beck, A. T., \& Steer, R. A. (1987). BDI, Beck depression inventory: Manual. New York: Psychological Corporation.

Beck, A. T., \& Steer, R. A. (1991). Manual for the Beck scale for suicide ideation. San Antonio, TX: Psychological Corporation.

Bergen, H., Hawton, K., Waters, K., Cooper, J., \& Kapur, N. (2010). Epidemiology and trends in non-fatal self-harm in three centres in England: 2000-2007. British Journal of Psychiatry, 197(6), 493-498. doi:10.1192/bjp.bp.110.077651

Brown, G. K. (2001). A review of suicide assessment measures for intervention research with adults and older adults. Retrieved 18 September 2014, http://ruralccp.org/lyradata/storage/asset/brown-nd-27cb.pdf

Brown, G. K., \& Jager-Hyman, S. (2014). Evidence-based psychotherapies for suicide prevention: Future directions. American Journal of Preventive Medicine, 47(3 Suppl 2), S186-S194. doi:10.1016/j.amepre.2014.06.008

Brown, G. K., Ten Have, T., Henriques, G. R., Xie, S. X., Hollander, J. E., \& Beck, A. T. (2005). Cognitive therapy for the prevention of suicide attempts: A randomized controlled trial. JAMA, 294(5), 563-570. doi:10.1001/jama.294.5.563

Bryan, C. J., Corso, K. A., Corso, M. L., Kanzler, K. E., Ray-Sannerud, B., \& Morrow, C. E. (2012). Therapeutic alliance and change in suicidal ideation during treatment in integrated primary care settings. Archives of Suicide Research, 16(4), 316-323. doi:10.1080/13811118.2013.722055 
Carroll, R., Metcalfe, C., \& Gunnell, D. (2014). Hospital presenting self-harm and risk of fatal and non-fatal repetition: Systematic review and meta-analysis. PLoS ONE, 9(2), e89944. doi:10.1371/journal.pone.0089944

Carter, G. L., Clover, K., Whyte, I. M., Dawson, A. H., \& D'Este, C. (2007). Postcards from the edge: 24-month outcomes of a randomised controlled trial for hospital-treated selfpoisoning. British Journal of Psychiatry, 191, 548-553. doi:10.1192/bjp.bp.107.038406

Carter, G. L., Clover, K., Whyte, I. M., Dawson, A. H., \& D'Este, C. (2013). Postcards from the edge: 5-year outcomes of a randomised controlled trial for hospital-treated self-poisoning. British Journal of Psychiatry, 202(5), 372-380. doi:10.1192/bjp.bp.112.112664

Cedereke, M., \& Ojehagen, A. (2005). Prediction of repeated parasuicide after 1-12 months. European Psychiatry, 20(2), 101-109. doi:10.1016/j.eurpsy.2004.09.008

Cooper, J., Kapur, N., Webb, R., Lawlor, M., Guthrie, E., Mackway-Jones, K., \& Appleby, L. (2005). Suicide after deliberate self-harm: A 4-year cohort study. American Journal of Psychiatry, 162(2), 297-303. doi:10.1176/appi.ajp.162.2.297

Crowder, R., Van der Putt, R., Ashby, C. A., \& Blewett, A. (2004). Deliberate self-harm patients who discharge themselves from the general hospital without adequate psychosocial assessment. Crisis, 25(4), 183-186. doi:10.1027/0227-5910.25.4.183

de Beurs, D. P., Fokkema, M., de Groot, M. H., de Keijser, J., \& Kerkhof, A. J. F. M. (2015). Longitudinal measurement invariance of the beck scale for suicide ideation. Psychiatry Research, 225(3), 368-373. doi:10.1016/j.psychres.2014.11.075

Fenton, L. R., Cecero, J. J., Nich, C., Frankforter, T. L., \& Carroll, K. M. (2001). Perspective is everything: the predictive validity of six working alliance instruments. Journal of Psychotherapy Practice and Research, 10(4), 262-268.

Fidy, R. (2008). Psychologische suizidalitäts-diagnostik im internet. Zürich: Universität Zürich.

Gaston, L., Thompson, L., Gallagher, D., Cournoyer, L., \& Gagnon, R. (1998). Alliance, technique, and their interactions in predicting outcome of behavioural, cognitive, and brief dynamic therapy. Psychotherapy Research, 8, 190-209. doi:10.1080/10503309812331332307

Granboulan, V., Roudot-Thoraval, F., Lemerle, S., \& Alvin, P. (2001). Predictive factors of postdischarge follow-up care among adolescent suicide attempters. Acta Psychiatrica Scandinavica, 104(1), 31-36. doi:10.1034/j.1600-0447.2001.00297.x

Hautzinger, M., Bailer, M., Worall, H., \& Keller, F. (1994). Beck-depressions-inventar (BDI). Bearbeitung der deutschen Ausgabe. Testhandbuch. Bern: Huber.

Haw, C., Bergen, H., Casey, D., \& Hawton, K. (2007). Repetition of deliberate self-harm: A study of the characteristics and subsequent deaths in patients presenting to a general hospital according to extent of repetition. Suicide and Life-Threatening Behavior, 37(4), 379-396. doi:10.1521/suli.2007.37.4.379

Heyerdahl, F., Bjornaas, M. A., Dahl, R., Hovda, K. E., Nore, A. K., Ekeberg, O., \& Jacobsen, D. (2009). Repetition of acute poisoning in Oslo: 1-year prospective study. British Journal of Psychiatry, 194(1), 73-79. doi:10.1192/bjp.bp.107.048322

Horesh, N., Levi, Y., \& Apter, A. (2012). Medically serious versus non-serious suicide attempts: Relationships of lethality and intent to clinical and interpersonal characteristics. Journal of Affective Disorders, 136(3), 286-293. doi:10.1016/j.jad.2011.11.035

Horrocks, J., Price, S., House, A., \& Owens, D. (2003). Self-injury attendances in the accident and emergency department: Clinical database study. British Journal of Psychiatry, 183, 34-39. doi:10.1192/bjp.183.1.34 
Horvath, A.O., Del Re, A. C., Flückiger, C., \& Symonds, D. (2011). Alliance in individual psychotherapy. Psychotherapy, 48(1), 9-16. doi:10.1037/a0022186

Horvath, A. O., Gaston, L., \& Luborsky, L. (1993). The therapeutic alliance and its measures. In L. L. N. Miller, J. Barber, \& J. P. Docherty (Eds.), Psychodynamic Treatment Research. New York: Basic Books.

Jenkins, G. R., Hale, R., Papanastassiou, M., Crawford, M. J., \& Tyrer, P. (2002). Suicide rate 22 years after parasuicide: Cohort study. BMJ, 325(7373), 1155. doi:10.1136/bmj.325.7373.1155

Jobes, D. A. (2000). Collaborating to prevent suicide: A clinical-research perspective. Suicide and Life-Threatening Behavior, 30(1), 8-17.

Kapur, N., Gunnell, D., Hawton, K., Nadeem, S., Khalil, S., Longson, D., ..., \& Cooper, J. (2013). Messages from Manchester: Pilot randomised controlled trial following self-harm. British Journal of Psychiatry, 203(1), 73-74. doi:10.1192/bjp.bp.113.126425

Kessler, R. C., Borges, G., \& Walters, E. E. (1999). Prevalence of and risk factors for lifetime suicide attempts in the national comorbidity survey. Archives of General Psychiatry, 56(7), 617-626. doi:10.1001/archpsyc.56.7.617

King, C. A., Hovey, J. D., \& Brand, E., et al. (1997). Suicidal adolescents after hospitalization: Parent and family impacts on treatment follow-through. Journal of the American Academy of Child and Adolescent Psychiatry, 36, 85-93. doi:10.1097/00004583-199701000-00021

Lizardi, D., \& Stanley, B. (2010). Treatment engagement: A neglected aspect in the psychiatric care of suicidal patients. Psychiatric Services, 61(12), 1183-1191. doi:10.1176/appi.ps.61.12.1183

Luborsky, L., Barber, J. P., Lynne, S., Johnson, S., Najavitis, L. M., Frank, A., \& Daley, D. (1996). The revised helping alliance questionnaire (HAQ-II): Psychometric properties. The Journal of Psychotherapy Practice and Research, 5, 260-271.

Maltsberger, J. T. (2004). The descent into suicide. The International Journal of Psychoanalysis, 85(3), 653-668. doi:10.1516/3c96-uret-tlwx-6lwu

Michel, K., Dey, P., Stadler, K., \& Valach, L. (2004). Therapist sensitivity towards emotional lifecareer issues and the working alliance with suicide attempters. Archives of Suicide Research, 8(3), 203-213. doi:10.1080/13811110490436792

Michel, K., \& Gysin-Maillart, A. (2015). Attempted suicide short intervention program ASSIP. A manual for clinicians. Göttingen: Hogrefe.

Michel, K., Maltsberger, J. T., Jobes, D. A., Leenaars, A. A., Orbach, I., Stadler, K., ..., \& Valach, L. (2002). Discovering the truth in attempted suicide. American Journal of Psychotherapy, 56(3), 424-437.

Michel, K., \& Valach, L. (1997). Suicide as goal-directed action. Archives of Suicide Research, 3(3), 213-221. doi:10.1080/13811119708258273

Michel, K., Valach, L., \& Waeber, V. (1994). Understanding deliberate self-harm: The patients' views. Crisis, 15(4), 172-178.

Monti, K., Cedereke, M., \& Ojehagen, A. (2003). Treatment attendance and suicidal behavior 1 month and 3 months after a suicide attempt: A comparison between two samples. Archives of Suicide Research, 7, 167-174. doi:10.1080/13811110301581

Morgan, R., Luborsky, L., Crits-Christoph, P., Curtis, H., \& Solomon, J. (1982). Predicting the outcomes of psychotherapy by the Penn helping alliance rating method. Archives of General Psychiatry, 39(4), 397-402. 
Motto, J. A., \& Bostrom, A. G. (2001). A randomized controlled trial of postcrisis suicide prevention. Psychiatric Services, 52(6), 828-833. doi:10.1176/appi.ps.52.6.828

National Action Alliance: A Prioritized Research Agenda: An Action Plan to Save Lives. (2014). American Journal of Preventive Medicine Supplement.

Owens, D., Horrocks, J., \& House, A. (2002). Fatal and non-fatal repetition of self-harm systematic review. The British Journal of Psychiatry, 181(3), 193-199. doi:10.1192/bjp.181.3.193

Pinninti, N., Steer, R. A., Rissmiller, D. J., Nelson, S., \& Beck, A. T. (2002). Use of the Beck scale for suicide ideation with psychiatric inpatients diagnosed with schizophrenia, schizoaffective, or bipolar disorders. Behaviour Research and Therapy, 40(9), 1071-1079. doi:10.1016/s0005-7967(02)00002-5

Pompili, M., Innamorati, M., Di Vittorio, C., Sher, L., Girardi, P., \& Amore, M. (2014). Sociodemographic and clinical differences between suicide ideators and attempters: A study of mood disordered patients 50 years and older. Suicide and Life-Threatening Behavior, 44(1), 34-45. doi:10.1111/sltb.12051

Rickham, P.P. (1964). Human experimentation. Code of ethics of the world medical association. Declaration of Helsinki. BMJ, 2(177). doi:10.1136/bmj.2.5402.177

Rogers, J. R., \& Soyka, K. M. (2004). “One size fits all”: An existential-constructivist perspective on the crisis intervention approach with suicidal individuals. Journal of Contemporary Psychotherapy, 34(1), 7-22. doi:10.1023/b:jocp.0000010910.74165.3a

Rudd, M. D., Bryan, C. J., Wertenberger, E. G., Peterson, A. L., Young-McCaughan, S., Mintz, J., .., \& Bruce, T. O. (2015). Brief cognitive-behavioral therapy effects on post-treatment suicide attempts in a military sample: Results of a randomized clinical trial with 2-year follow-up. American Journal of Psychiatry, 172, 441-449. doi:10.1176/appi.ajp.2014.14070843

Runeson, B., Tidemalm, D., Dahlin, M., Lichtenstein, P., \& Langstrom, N. (2010). Method of attempted suicide as predictor of subsequent successful suicide: national long term cohort study. BMJ, 341, c3222. doi:10.1136/bmj.c3222

Sakinofsky, I. (2000). Repetition of suicidal behaviour. In K. Hawton \& K. Van Heeringen (Eds.), The international handbook of suicide and attempted suicide (pp. 385-404). Chichester: Wiley.

Saltzman, C., Luetgert, M. J., Roth, C. H., Creaser, J., \& Howard, L. (1976). Formation of a therapeutic relationship: Experiences during the initial phase of psychotherapy as predictors of treatment duration and outcome. Journal of Consulting and Clinical Psychology, 44(4), 546-555. doi:10.1037/0022-006x.44.4.546

Shneidman, E. S. (1993). Commentary: Suicide as psychache. The Journal of Nervous and Mental Disease, 181(3), 145-147. doi:10.1097/00005053-199303000-00001

Silverman, M. M., Berman, A. L., Sanddal, N. D., O'Carroll, P. W., \& Joiner, T. E. (2007). Rebuilding the tower of Babel: A revised nomenclature for the study of suicide and suicidal behaviors. Part 2: Suicide-related ideations, communications, and behaviors. Suicide and Life-Threatening Behavior, 37(3), 264-277. doi:10.1521/suli.2007.37.3.264

Steer, R. A., Rissmiller, D. J., Ranieri, W. F., \& Beck, A. T. (1993). Dimensions of suicidal ideation in psychiatric inpatients. Behaviour Research and Therapy, 31(2), 229-236. doi:10.1016/0005-7967(93)90090-h

Treolar, A. J., \& Pinfold, T. J. (1993). Deliberate self-harm: An assessment of patients' attitudes to the care they receive. Crisis, $14,83-89$. 
WHO (2014). The ICD-10 classification of mental and behavioral disorders: Clinical descriptions and diagnostic guidelines. Geneva: WHO.

Young, R. A., \& Valach, L. (2002). The self-confrontation interview in suicide research. Lifenotes, 7, 12-14. 
Table 1. Demographic and clinical characteristics

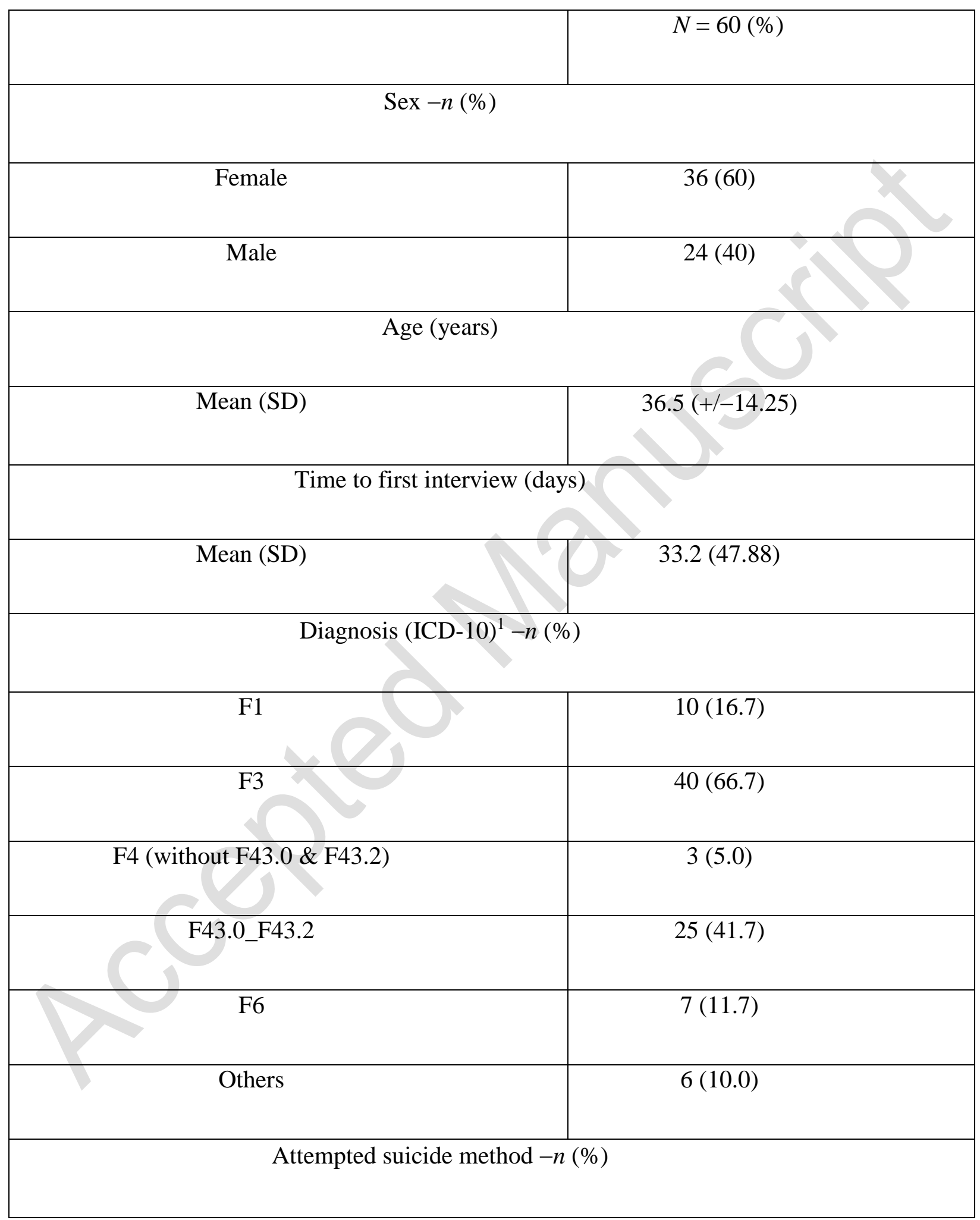




\begin{tabular}{|c|c|}
\hline Overdose & $38(63.3)$ \\
\hline Cutting & $8(13.3)$ \\
\hline Jumping & $5(8.3)$ \\
\hline Other (hanging, shooting, drowning) & \\
& \\
& \\
\hline & \\
\hline & \\
\hline & \\
\hline & \\
\hline & \\
\hline & \\
\hline
\end{tabular}

${ }^{1}$ F1: Substance related disorders; F3: affective disorders; F4: neurotic, stress-related, and somatoform disorders; F43.0 \&

F43.2: acute stress reaction, adjustment disorder; F6: personality disorder; others: F5, Behavioral syndromes with physical disorder and F9, Disorders usually first diagnosed in infancy, childhood, or adolescence. 
Table 2. Spearman Correlation between suicidal ideation at $t 2$ and $t 3$, therapeutic alliance, baseline scores of depressive symptoms and previous suicide attempts.

\begin{tabular}{|c|l|l|}
\hline & BSS $t 2(N=60)$ & BSS $t 3(N=60)$ \\
\hline HAQ1 & $r=-0.47 ; p=.000$ & $r=-0.50 ; p=.000$ \\
\hline HAQ2 & $r=-0.32 ; p=.013$ & $r=-0.36 ; p=.005$ \\
\hline BDI baseline & $r=0.49 ; p=.000$ & $r=0.40 ; p=.002$ \\
\hline PSA & $r=0.59 ; p=.000$ & $r=0.56 ; p=.000$ \\
\hline
\end{tabular}

BSS: Beck Scale for Suicide Ideation (Beck \& Steer, 1991); BSSt2: 6 months follow up; BSSt3: 12 months follow-up.

HAq: Therapeutic Alliance Questionnaire (Alexander \& Luborsky, 1986); HAq1: first therapy session; HAq2: third therapy session; BDI: Beck Depression Inventory (Beck \& Steer, 1987), BDI Baseline: first therapy session; PSA:

Previous Suicide Attempts. 
Table 3. Stepwise multiple linear regression analysis of suicidal ideation (BSSt3) at 12 months follow-up ${ }^{\text {. }}$.

\begin{tabular}{|c|c|c|c|c|c|c|c|c|}
\hline Variable & $R^{2}$ & Corrected & $\Delta R^{2}$ & $\Delta F$ & $\Delta P$ & Standardized $\beta$ & $T$ & $P$ \\
\hline Step 1 & .325 & .301 & .325 & 13.705 & .000 & & & \\
\hline BDIt1 & & & & & & .153 & 1.200 & .235 \\
\hline PSA & & & & & & .475 & 3.725 & .000 \\
\hline Step 2 & .417 & .386 & .093 & 8.892 & .004 & & & \\
\hline HAq1 & & & & & & -.334 & -2.982 & .004 \\
& & & & & & & & \\
\hline
\end{tabular}

a Model: $F=20.17, \mathrm{df}=2,59, p=.000$.

BSS: Beck Scale for Suicide Ideation (Beck \& Steer, 1991, BSSt3: 12 months follow-up); BDIt1: Beck Depression Inventory (Beck \& Steer, 1987), first therapy session; PSA: Previous Suicide Attempts; HAq: Therapeutic Alliance Questionnaire (Alexander \& Luborsky, 1986); HAq1: first therapy session. 